Regular Article

Tighlighted Leaper selected by Bditor-in-Ghief

\title{
Preparation of Cocoa Powder-Containing Orally Disintegrating Tablets of Rebamipide (Rebamipide Chocolet) and Evaluation of Their Clinical Palatability
}

\author{
Hidehito Takano, ${ }^{a, b}$ Shinya Uchida, ${ }^{*, a}$ Yasuharu Kashiwagura, ${ }^{a}$ Shimako Tanaka, ${ }^{a}$ \\ Akio Hakamata, ${ }^{c}$ Keiichi Odagiri,${ }^{c, d}$ Naoki Inui, ${ }^{c}$ Hiroshi Watanabe,${ }^{c}$ and Noriyuki Namiki ${ }^{a}$ \\ ${ }^{a}$ Department of Pharmacy Practice and Science, School of Pharmaceutical Sciences University of Shizuoka; \\ 52-1 Yada, Suruga-ku, Shizuoka 422-8526, Japan: ${ }^{b}$ Department of Pharmacy, Kameda Medical Center Hospital; \\ 929 Highashi-cho, Kamogawa, Chiba 296-8602, Japan: ${ }^{c}$ Department of Clinical Pharmacology \& Therapeutics, \\ Hamamatsu University School of Medicine; 1-20-1 Handayama, Higashi-ku, Hamamatsu 431-3192, Japan: and \\ ${ }^{d}$ Center for Clinical Research, Hamamatsu University Hospital; 1-20-1 Handayama, Higashi-ku, Hamamatsu 431- \\ 3192, Japan.
}

Received August 15, 2018; accepted November 12, 2018

Orally disintegrating tablets (ODTs), which are administered without water, are beneficial for elderly patients and patients with dysphagia. Masking the unpleasant taste of a drug is an important factor associated with adherence of patients consuming ODTs. We prepared cocoa powder-containing ODTs of bittertasting rebamipide (rebamipide chocolet) and evaluated their clinical palatability. We prepared rebamipide ODTs by adding a sweetener and $0,2.5,5$, and $10 \%$ cocoa powder (Ch0-ODTs, Ch2.5-ODTs, Ch5-ODTs, and Ch10-ODTs, respectively). Rebamipide ODTs without cocoa powder and sweetener were used as controls (Cont-ODTs). We performed a gustatory sensation test in 30 healthy adult volunteers. We used the 100-mm visual analog scale (VAS) to evaluate bitterness, sweetness, scent, and overall palatability of the ODTs. The acceptability of each ODT was evaluated on a 5-point scale. Compared to Cont-ODTs, Ch0-ODTs showed no significant improvement in the VAS score for bitterness, scent, and overall palatability during disintegration. However, compared to Cont-ODTs, Ch2.5-ODTs, Ch5-ODTs, and Ch10-ODTs showed an improvement in all items evaluated using the VAS. In particular, Ch2.5-ODTs showed a significant improvement compared to the Cont-ODTs in the VAS score of all items. Evaluation on a 5-point scale indicated that Ch2.5-ODTs and Ch10-ODTs had the highest acceptability. We prepared rebamipide chocolet with excellent palatability properties, which could not be achieved using a sweetener alone, by using the combination of a sweetener and cocoa powder as a new agent for masking bitterness. Our results indicate that cocoa powder may be used as a taste-masking agent in ODTs.

Key words orally disintegrating tablet; rebamipide; cocoa powder; chocolet; bitterness masking agent; gustatory sensation test

\section{Introduction}

Tablets are the most widely used pharmaceutical dosage form because they are easy to handle and convenient to carry and store. However, tablets cannot be administered to patients with decreased swallowing ability, such as elderly patients and children. ${ }^{1-4)}$ In addition, consuming tablets is a burden for patients with kidney disease with restriction of water intake and for patients with dysuria who avoid drinking water. Tablets are associated with poor patient adherence, and thus, the expected therapeutic effect of tablets cannot be obtained; the issue of poor adherence needs to be resolved to achieve effective drug therapy. Orally disintegrating tablets (ODTs), which disintegrate quickly and are easily swallowed with a small amount of water in the oral cavity, have been introduced clinically and can be easily consumed by patients with decreased swallowing ability or those with kidney disease and dysuria. ${ }^{5)}$ In addition, ODTs can be taken regardless of the location of patients thus improving medication adherence. Thus, ODTs are gaining increasing popularity worldwide. ${ }^{\text {) }}$

Rebamipide protects the gastric mucosa, and it is widely used in patients with gastric ulcer and gastritis, including elderly patients. Administration of non-steroidal anti-inflam- matory drugs (NSAIDs) and steroids is associated with the development of peptic ulcers ${ }^{7-9}$; ; aspirin increases the risk of gastrointestinal bleeding even at low doses. ${ }^{6,10)}$ Many elderly patients are prescribed NSAIDs and steroids, and development of ulcers subsequent to the consumption of these drugs necessitates treatment with drugs such as rebamipide. Thus, ODTs of rebamipide are highly useful for patients who have difficulty in swallowing tablets. One of the biggest challenge in developing an ODT of rebamipide is overcoming the strong bitterness of this drug. ODTs disintegrate in the oral cavity, and thus, bitterness of a drug can significantly lower medication adherence, thereby decreasing the therapeutic effect. Therefore, masking the bitterness of the drug is important.

Bitterness of a drug can be masked using various methods. One of the taste-masking method involves suppressing the bitterness of a drug by modifying its organoleptic properties by adding a flavoring substance or a bitter blocker to the preparation. $^{11-13)}$ The abovementioned method is the most convenient taste-masking method. Cocoa has been used as a flavoring agent in a variety of food and confectionery items. Clinically, cocoa powder has been used as an effective ingredient for masking the bitterness of medicines, particularly of pediatric 
medicines. However, no studies to date have reported the effectiveness of cocoa powder in masking the bitter taste of drugs that are being formulated as ODTs.

This study aimed to develop an ODT containing cocoa powder, named as "Chocolet," for masking the bitterness of the drug. We prepared rebamipide chocolet and evaluated the effect of cocoa powder on the palatability of rebamipide chocolet using gustatory sensation tests in healthy volunteers.

\section{Experimental}

Materials Rebamipide was provided by Otsuka Pharmaceutical Co., Ltd. (Tokyo, Japan). All other samples were commercially obtained as follows: light anhydrous silicic acid (Adsolider 101; Freund Corp., Tokyo, Japan), pregelatinized starch (SWELSTAR WB-1; Asahi Kasei Corp., Tokyo, Japan), mannitol (Pearlitol Flash; Roquette Japan, Tokyo, Japan), Ludiflash (BASF Japan Ltd., Tokyo, Japan), crospovidone (Kollidon CL-SF; BASF), sodium stearyl fumarate (Wako Pure Chemical Industries, Ltd., Corp., Osaka, Japan), aspartame (Ajinomoto Co., Inc., Tokyo, Japan), thaumatin (San-Ei Gen F.F.I., Inc., Toyonaka, Japan), and cocoa powder (NF-15; Morinaga Shoji Co., Ltd.). The commercial product of rebamipide (Mucosta tablet $100 \mathrm{mg}$ ) was purchased from Otsuka Pharmaceutical Co., Ltd. All raw materials used in this study were Japanese Pharmacopeia (JP) 17th edition (JP17) compliant products, and the reagents used were either listed in the JP or commercial special grade chemicals.

Preparation of Rebamipide Granules Rebamipide $(600 \mathrm{~g})$ and light anhydrous silicic acid $(12.0 \mathrm{~g})$ were mixed. Pregelatinized starch (7.5 g) and mannitol (225.0 g) were weighed and dissolved in purified water to prepare $2000 \mathrm{~g}$ of a binding solution. The mixture of starch and mannitol $(1500 \mathrm{~g})$ was granulated using a fluidized-bed granulator (MP-01; Powrex Corp., Hyogo, Japan) and was sprayed on rebamipide and light anhydrous silicic acid. The binding solution was sprayed at a rate of $15 \mathrm{~mL} / \mathrm{min}$ during the first $30-45 \mathrm{~min}$ and then at a rate of $10 \mathrm{~mL} / \mathrm{min}$. The parameters at the time of spraying were intake air temperature $70^{\circ} \mathrm{C}$, air flow rate $30-40 \mathrm{~m}^{3} / \mathrm{h}$, bottom stirring $500 \mathrm{rpm}$, spray air pressure 2 bar, and spray air flow rate $35 \mathrm{NL} / \mathrm{min}$. After granulation, the granules were dried using a fluidized bed dryer and were sieved using a $355-\mu \mathrm{m}$ mesh; the granules recovered after sieving were used as rebamipide granules.

Preparation of Rebamipide ODTs The formulation of rebamipide ODTs is shown in Table 1. Ludiflash was used as an excipient for preparing rebamipide ODTs. In addition, $10 \%$ of crospovidone was added as a disintegrating agent and
$0.5 \%$ of sodium stearyl fumarate as a lubricant. Rebamipide granules were formulated to contain rebamipide $100 \mathrm{mg}$ per tablet (Cont-ODTs). Rebamipide ODTs-containing sweeteners were prepared by adding $1 \%$ each of aspartame and thaumatin to Cont-ODT (Ch0-ODTs). Rebamipide chocolet was prepared by adding cocoa powder to Ch0-ODT. Three kinds of rebamipide chocolet were prepared by adding $2.5,5$, and $10 \%$ of cocoa powder NF-15 to Ch0-ODT (Ch2.5-ODT, Ch5-ODT, and Ch10-ODT, respectively). The quantity of Ludiflash was adjusted to achieve a final tablet weight of $350 \mathrm{mg}$.

The ODTs, $12 \mathrm{~mm}$ in diameter, were prepared using a tablet compressor (HANDTAB-100; Ichihashi Seiki Co., Ltd., Kyoto, Japan) by direct compression to achieve a tablet hardness of about $50 \mathrm{~N}$. The addition of sodium stearyl fumarate to the formulation containing cocoa powder NF-15 for preventing tableting problems was unnecessary.

Contents of rebamipide in the ODTs were measured using HPLC system (LC-2010; Shimadzu, Kyoto, Japan) comprising a packed column (TSK gel ODS-100V; $5 \mu \mathrm{m}, 4.6 \mathrm{~mm}$ i.d. $\times 150 \mathrm{~mm}$; TOSOH, Tokyo, Japan). The mobile phase was phosphate buffer $(14 \mathrm{mM})$ /acetonitrile $(83 / 17, \mathrm{v} / \mathrm{v})$. The flow rate was $1 \mathrm{~mL} / \mathrm{min}$ and detection was based on UV absorbance at $254 \mathrm{~nm}$.

Hardness of the ODTs The hardness of the ODTs was measured using a load cell-type tablet hardness scale (PC-30; Okada Seiko Co., Ltd., Tokyo, Japan). The hardness of 10 tablets of each type of ODT was measured and the mean hardness was calculated.

In Vitro Disintegration Time of the ODTs Disintegration time of the ODTs in vitro was measured using Tricorptester (Okada Seiko Co., Ltd., Japan). The test saliva $(\mathrm{NaCl}$, $1.44 \mathrm{~g} / \mathrm{L} ; \mathrm{KCl}, 1.47 \mathrm{~g} / \mathrm{L}$; and Tween $80,0.3 \%$ ) warmed to $37^{\circ} \mathrm{C}$ was used as the test solution and was dripped from a height of $80 \mathrm{~mm}$ at a flow rate of $6.0 \mathrm{~mL} / \mathrm{min}$. The measurement was performed using 10 tablets of each type of ODT, and the mean disintegration time was calculated.

Tablet Friability Tablet friability was evaluated using a tablet friabilator (EF-1W; Electrolab, Mumbai, India). A number of tablets were weighed and placed in the drum, where they were rotated and exposed to repeated shocks. The weight of all tablets remaining in the drum after 100 revolutions was measured, and the difference in the weight before and after rotation was calculated.

Dissolution Test The dissolution test was performed in accordance with the JP17 dissolution test method 2 (paddle method) using a dissolution tester (PJ-6S; Miyamoto Riken Ind. Co., Ltd., Osaka, Japan). The tests for Cont-ODTs, Ch0-

Table 1. Formulation of Rebamipide ODTs

\begin{tabular}{|c|c|c|c|c|c|}
\hline Component (\%) & Cont-ODT & Ch0-ODT & $\mathrm{Ch} 2.5$-ODT & Ch5-ODT & Ch10-ODT \\
\hline Rebamipide granules & 41.6 & 41.6 & 41.6 & 41.6 & 41.6 \\
\hline Ludiflash & 47.9 & 45.9 & 43.9 & 41.4 & 36.4 \\
\hline Crospovidone & 10 & 10 & 10 & 10 & 10 \\
\hline Aspartame & 0 & 1.0 & 1.0 & 1.0 & 1.0 \\
\hline Thaumatin & 0 & 1.0 & 1.0 & 1.0 & 1.0 \\
\hline Cocoa powder (NF-15) & 0 & 0 & 2.5 & 5.0 & 10.0 \\
\hline Sodium stearyl fumarate & 0.5 & 0.5 & 0 & 0 & 0 \\
\hline Total $(\%)$ & 100.0 & 100.0 & 100.0 & 100.0 & 100.0 \\
\hline
\end{tabular}

Cont-ODTs, rebamipide orally disintegrating tablets without cocoa powder and sweetener; Ch0-ODTs, Ch2.5-ODTs, Ch5-ODTs, and Ch10-ODTs, rebamipide ODTs prepared by adding a sweetener and $0,2.5,5$, and $10 \%$ cocoa powder, respectively. 


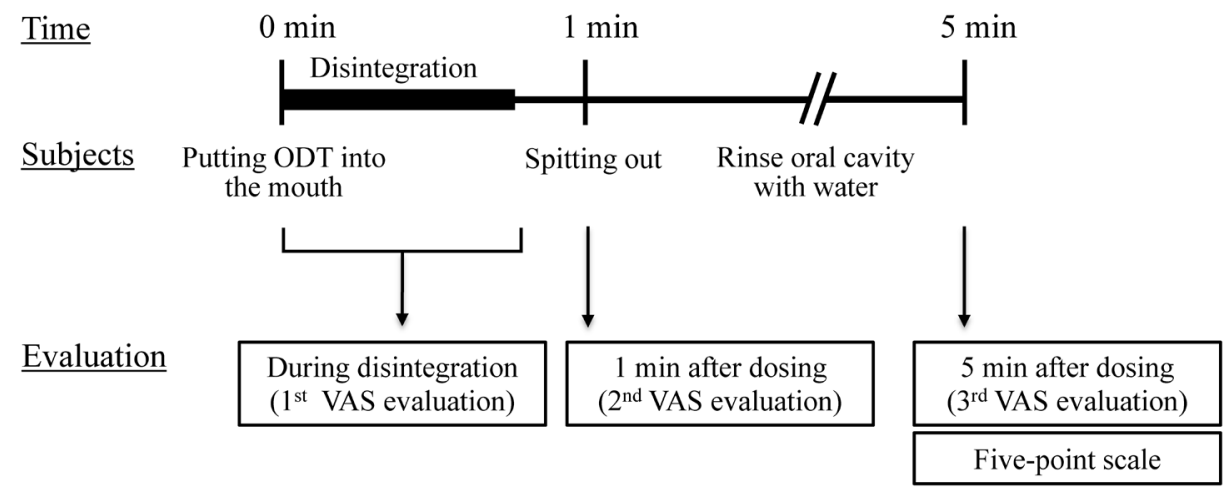

Fig. 1. Time Schedule of the Gustatory Sensation Test in Healthy Volunteers

ODTs, Ch2.5-ODTs, Ch5-ODTs, Ch10-ODTs, and a commercial product (Mucosta tablet, Otsuka Pharmaceutical Co., Ltd.) were performed using Mcllvaine buffer ( $\mathrm{pH}$ 5.5) and a JP17 second fluids ( $\mathrm{pH}$ 6.8) as a dissolution medium with paddle speed of $37 \pm 0.5^{\circ} \mathrm{C}$ at $50 \mathrm{rpm}$ for one tablet. Samples of the dissolved solution with the buffer of $\mathrm{pH} 5.5$ were taken at $0,5,10,15,20,30,45,60,120,240$, and $360 \mathrm{~min}$ and those containing buffer $\mathrm{pH} 6.8$ were taken at $0,5,10,15,20$, and $30 \mathrm{~min}$. The concentration of rebamipide in the samples was determined using HPLC at a wavelength of $326 \mathrm{~nm}$.

Measurement of the Gustatory Sensation of ODTs in Healthy Volunteers Using a Visual Analog Scale and a 5-Point Rating Scale The gustatory sensation test was performed to evaluate the palatability of ODTs. The test was conducted in accordance with the Declaration of Helsinki and its amendments. The protocol was approved by the Ethics Committee of the Hamamatu University Hospital (Shizuoka, Japan). All subjects provided written consent to participate in the test.

This study was designed by modifying part of the methods detailed by Nakano et al. ${ }^{11)}$ and Matsui et al. ${ }^{12)}$ (Fig. 1). We enrolled 30 healthy volunteers ( 9 men and 21 women; age, $22.3 \pm 2.2$ years, mean \pm standard deviation (S.D.)) in a randomized crossover single-blind study. The subjects were randomly assigned to a group of ODTs (Cont-ODT, Ch0-ODT, Ch2.5-ODT, Ch5-ODT, and Ch10-ODT) according to the Latin square rating method. The subjects rinsed their mouth with water $5 \mathrm{~min}$ before commencing the test. The subjects placed the ODT in the oral cavity after a cue from the examiner and disintegrated the ODT. The subjects evaluated the overall palatability as well as the bitterness, scent, and sweetness of the ODT using a visual analogue scale (VAS). All subjects were asked to place a mark along the VAS line. The strongest sensation for each parameter was marked at $100 \mathrm{~mm}$. The first evaluation was from placement of one ODT in the mouth to completion of disintegration (During disintegration). The subject held the ODT in the oral cavity without swallowing and spat out the disintegrated tablet $1 \mathrm{~min}$ after dosing. Then, they immediately conducted the second evaluation. Subsequently, the subjects rinsed the residues in the oral cavity with $120 \mathrm{~mL}$ of water. The third evaluation was conducted 5 min after dosing. Measurement of the clinical disintegration time of each ODT in the oral cavity was performed using a stopwatch simultaneously with VAS evaluation.

After the third evaluation, the acceptability of the ODT was evaluated using a 5-point scale. The 5-point score for evaluat- ing acceptability was as follows: 1 , extremely accepted; 2 , accepted; 3, neither; 4, unacceptable; 5 , extremely unacceptable. The interval to the next protocol was secured for $30 \mathrm{~min}$.

Receiver Operating Characteristic to Determine the Relation between VAS Scores and the Acceptability of the ODTs To evaluate the relationship between VAS scores and the acceptability of the ODT, we performed receiver operating characteristic (ROC) curve analysis. The ROC curve is a plot of the sensitivity and specificity across the range of residency size cut-off points derived from $2 \times 2$ metrics. The ODTs with a score 1-3 on the 5-point scale were defined as "acceptable." On the basis of the ROC curve, we calculated the threshold VAS scores (cut-off values) of overall palatability at each evaluation for the acceptability the ODT on a 5-point rating scale, and the area under the curve $(A U C)$ values as an effective measure of accuracy. ROC curve analysis was conducted using JMP (ver. 8, SAS Institute Inc., Cary, NC, U.S.A.).

Statistical Analyses Data are expressed as mean \pm S.D. Statistical analysis was performed using paired $t$-test with post-hoc Bonferroni correction using GraphPad Prism software (version 5.0; GraphPad-San Diego, CA, U.S.A.). A statistically significant difference was noted at $p<0.0125$.

\section{Results}

Tablet Characteristics and Dissolution of ODTs The characteristics of rebamipide ODTs are shown in Table 2. An increase in the amount of cocoa powder necessitated an increase in compression force from 4 to $14 \mathrm{kN}$ to achieve the target hardness of $50 \mathrm{~N}$. The ODTs prepared had a hardness of 49.0 to $58.2 \mathrm{~N}$ and an in vitro disintegration time of 15.3 to $26.6 \mathrm{~s}$. Although the in vitro disintegration times showed an increasing trend with an increase in the amount of cocoa powder, they were within the target time of $30 \mathrm{~s}$. The contents of rebamipide and friability of Cont-ODT, Ch0-ODT, and Ch10ODT were $102.8-103.9$ and $<0.4 \%$, which conformed to the criteria according to JP 17.

The dissolution behavior of rebamipide was almost the same as that of the commercial product (Mucosta tablet) in all test solutions of $\mathrm{pH} 5.5$ and $\mathrm{pH}$ 6.8, which indicated no difference in the dissolution properties of both products (Fig. 2).

Clinical Disintegration Time of ODTs The clinical disintegration times of Cont-ODT, Ch0-ODT, Ch2.5-ODT, Ch5ODT, and Ch10-ODT were 26.2, 27.7, 27.6, 26.8, and $31.3 \mathrm{~s}$, respectively. The disintegration time of each ODT was around $30 \mathrm{~s}$, and no difference was observed between the different formulations. 
Table 2. Tablet Characteristics of Rebamipide ODTs

\begin{tabular}{lccccc}
\hline \hline & Cont-ODT & Ch0-ODT & Ch2.5-ODT & Ch5-ODT & Ch10-ODT \\
\hline Compression force $(\mathrm{kN})$ & 4 & 4 & 8 & 10 & 14 \\
\hline Hardness $(\mathrm{N})$ & $58.2 \pm 5.4$ & $55.0 \pm 3.0$ & $55.7 \pm 4.9$ & $49.0 \pm 4.8$ & $56.3 \pm 3.7$ \\
In vitro disintegration time (s) & $16.0 \pm 0.7$ & $15.3 \pm 0.3$ & $18.4 \pm 1.0$ & $20.3 \pm 2.4$ & $26.6 \pm 0.7$ \\
Friability (\%) & 0.4 & 0.4 & - & - & 0.28 \\
\hline
\end{tabular}

Hardness and in vitro disintegration times are shown as the mean \pm standard deviation (S.D.) ( $n=3-10)$. Data of friability are shown as \% of weight loss in one determination. Cont-ODTs, rebamipide orally disintegrating tablets without cocoa powder and sweetener; Ch0-ODTs, Ch2.5-ODTs, Ch5-ODTs, and Ch10-ODTs, rebamipide ODTs prepared by adding a sweetener and $0,2.5,5$, and $10 \%$ cocoa powder, respectively.

(A)

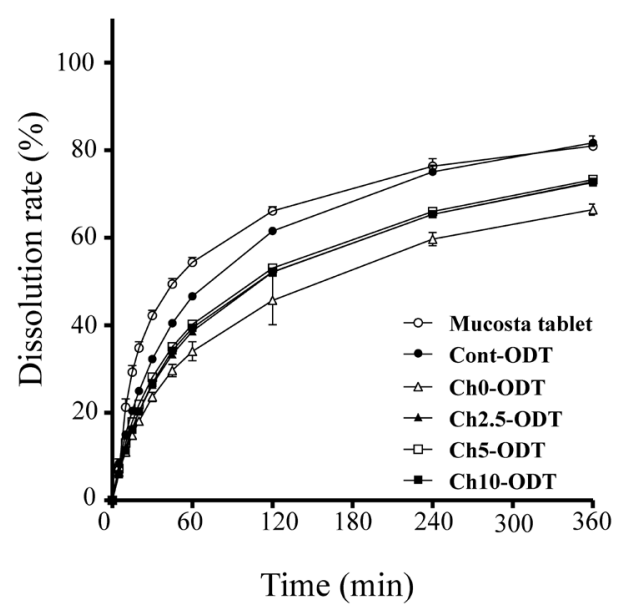

(B)

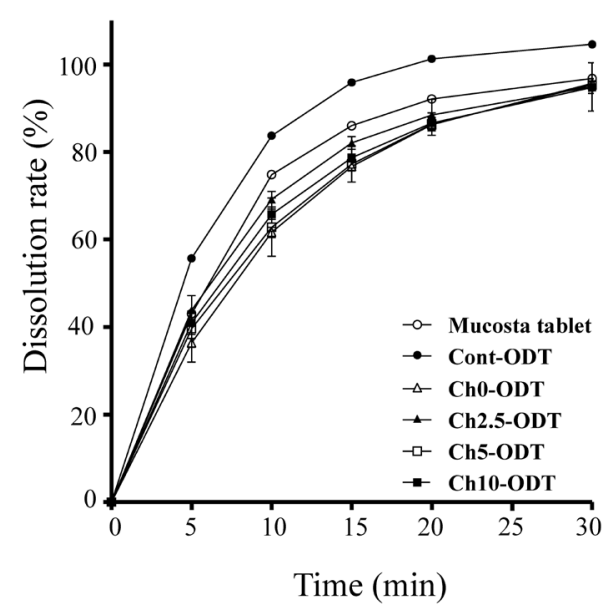

Fig. 2. Dissolution Profiles of Rebamipide from Cont-ODTs, Ch0-ODTs, Ch2.5-ODTs, Ch5-ODTs, Ch10-ODTs, and Mucosta Tablet in Mcllvaine Buffer (A, pH 5.5) and a JP17 Second Fluid (B, pH 6.8)

Cont-ODTs, rebamipide orally disintegrating tablets without cocoa powder and sweetener; Ch0-ODTs, Ch2.5-ODTs, Ch5-ODTs, and Ch10-ODTs, rebamipide ODTs prepared by adding a sweetener and $0,2.5,5$, and $10 \%$ cocoa powder, respectively. Each point represents the mean \pm S.D. $(n=6-12)$.

(A)

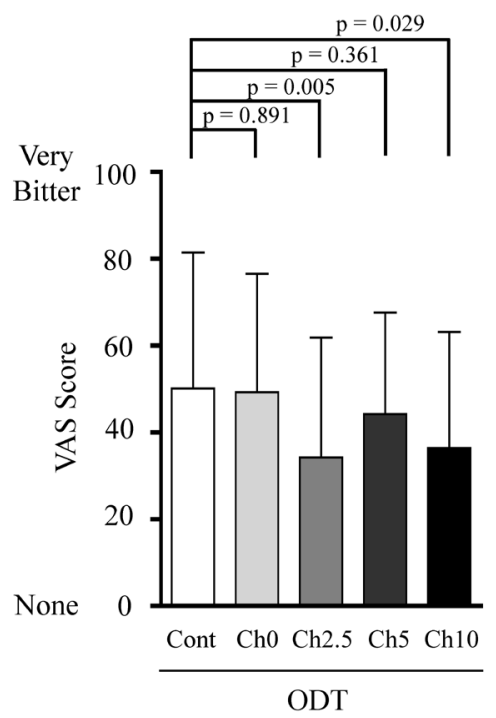

(B)

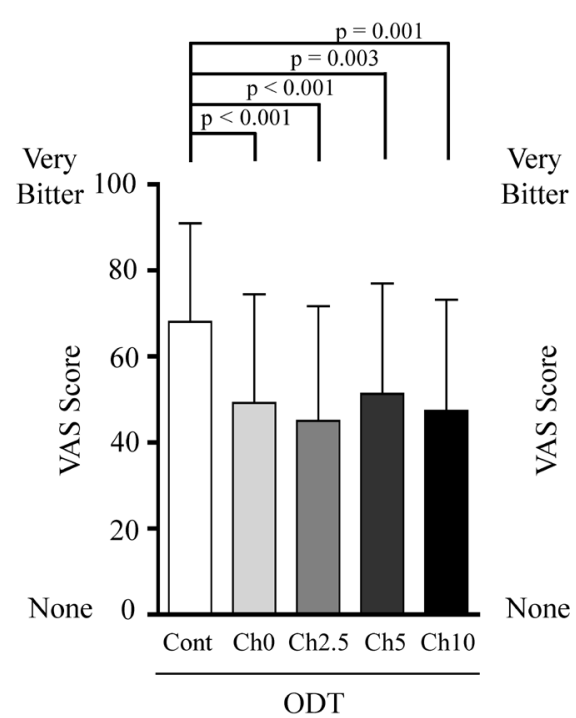

(C)

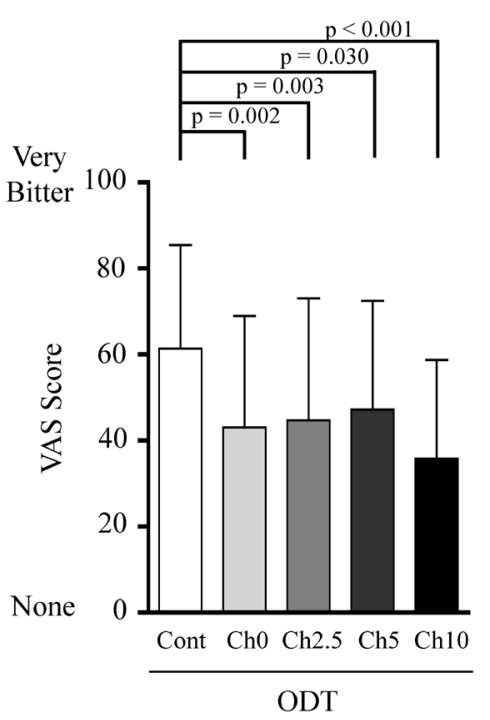

Fig. 3. The Visual Analog Scale (VAS) Scores for Bitterness During Disintegration (A), at 1 min (B), and 5 min (C) after Dosing of the Orally Disintegrating Tablets (ODTs) in the Gustatory Sensation Test

Each column represents the mean \pm S.D. $(n=30)$. Paired $t$-test with post-hoc Bonferroni correction was used to detect significant differences between the Cont-ODTs and the Ch0-ODTs, Ch2.5-ODTs, Ch5-ODTs, and Ch10-ODTs. A statistically significant difference was noted at $p<0.0125$. Cont-ODTs, rebamipide orally disintegrating tablets without cocoa powder and sweetener; Ch0-ODTs, Ch2.5-ODTs, Ch5-ODTs, and Ch10-ODTs, rebamipide ODTs prepared by adding a sweetener and 0, 2.5, 5, and $10 \%$ cocoa powder, respectively. 
Table 3. Visual Analog Scale (VAS) Scores for Scent and Sweetness in the Gustatory Sensation Test

\begin{tabular}{lccccc}
\hline \hline Evaluation & Cont-ODT & Ch0-ODT & Ch2.5-ODT & Ch5-ODT & Ch10-ODT \\
\hline Scent & & & & & \\
During disintegration & $11.0 \pm 21.0$ & $16.0 \pm 22.4$ & $35.8 \pm 28.2^{*}$ & $37.4 \pm 24.5^{*}$ & $49.0 \pm 32.6^{*}$ \\
1 min after dosing & $8.7 \pm 18.3$ & $13.3 \pm 18.2$ & $33.1 \pm 25.4^{*}$ & $30.9 \pm 25.2^{*}$ & $34.3 \pm 25.5^{*}$ \\
5 min after dosing & $3.1 \pm 10.6$ & $4.7 \pm 8.9$ & $9.2 \pm 17.6$ & $7.0 \pm 10.0$ & $12.2 \pm 17.6^{*}$ \\
Sweetness & & & & \\
During disintegration & $8.8 \pm 18.6$ & $19.3 \pm 22.2$ & $34.6 \pm 23.8^{*}$ & $29.5 \pm 24.4^{*}$ & $39.1 \pm 22.5^{*}$ \\
1 min after dosing & $3.0 \pm 5.1$ & $20.4 \pm 18.5^{*}$ & $27.5 \pm 22.0^{*}$ & $19.6 \pm 19.9^{*}$ & $31.8 \pm 20.2^{*}$ \\
5 min after dosing & $2.6 \pm 10.3$ & $8.4 \pm 13.2$ & $7.3 \pm 14.0$ & $5.0 \pm 7.5$ & $10.9 \pm 16.5^{*}$ \\
\hline
\end{tabular}

Data are shown as the mean \pm S.D. $(n=30)$. Asterisks denote a significant difference in values of Ch0-ODT, Ch2.5-ODT, Ch5-ODT, Ch10-ODT compared to Cont-ODT using the paired $t$-test with post-hoc Bonferroni correction, ${ }^{*} p<0.0125$. Cont-ODTs, rebamipide orally disintegrating tablets without cocoa powder and sweetener; Ch0-ODTs, Ch2.5-ODTs, Ch5-ODTs, and Ch10-ODTs, rebamipide ODTs prepared by adding a sweetener and $0,2.5,5$, and $10 \%$ cocoa powder, respectively.

(A)

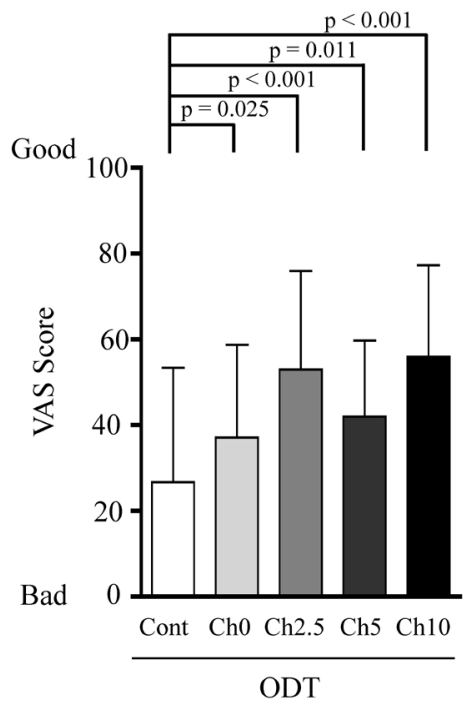

(B)

(C)

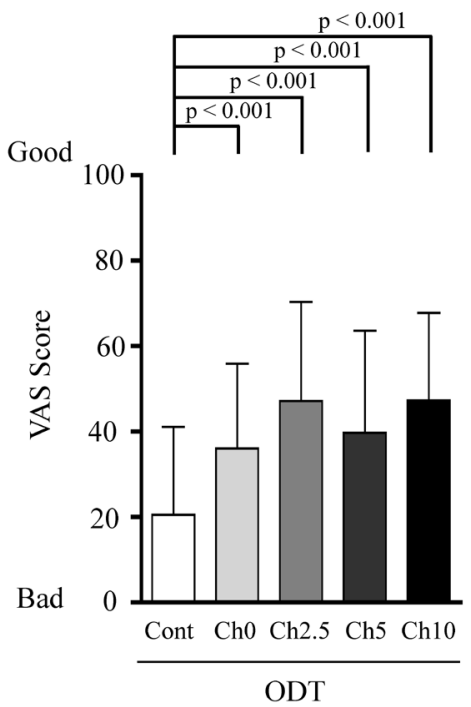

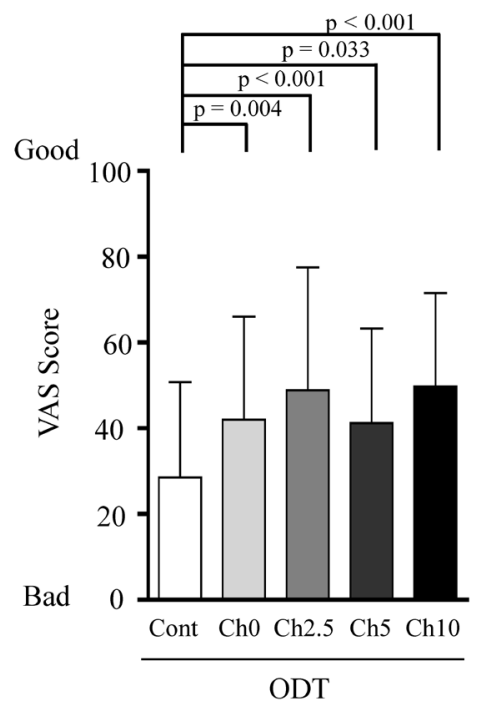

Fig. 4. The Visual Analog Scale (VAS) Scores for Overall Palatability During Disintegration (A), at 1 min (B), and 5 min (C) after Dosing of the Orally Disintegrating Tablets (ODTs) in the Gustatory Sensation Test

Each column represents the mean \pm S.D. $(n=30)$. Paired $t$-test with post-hoc Bonferroni correction was used to detect significant differences between Cont-ODTs and Ch0-ODTs, Ch2.5-ODTs, Ch5-ODTs, and Ch10-ODTs. A statistically significant difference was noted at $p<0.0125$. Cont-ODTs, rebamipide orally disintegrating tablets without cocoa powder and sweetener; Ch0-ODTs, Ch2.5-ODTs, Ch5-ODTs, and Ch10-ODTs, rebamipide ODTs prepared by adding a sweetener and 0, 2.5, 5, and 10\% cocoa powder, respectively.

VAS Score for Bitterness of ODTs The VAS score for bitterness of Cont-ODTs was 50.1 during disintegration, and the VAS score increased to 68.1 and 61.4 at 1 and $5 \mathrm{~min}$ after dosing (Fig. 3). The VAS score for bitterness during disintegration of Ch0-ODT was 49.3, which was similar to that of the Cont-ODT (Fig. 3A). Compared to the Cont-ODTs, the Ch2.5ODTs, Ch5-ODTs, and Ch10-ODTs showed a decrease in the VAS scores to 34.2, 44.2, and 36.4, respectively (Fig. 3A). In addition, the VAS scores for bitterness $1 \mathrm{~min}$ after dosing the Ch0-ODTs, Ch2.5-ODTs, Ch5-ODTs and Ch10-ODTs, were significantly lower than those of the Cont-ODT (Fig. 3B), and the VAS scores of ODTs continued to decrease $5 \mathrm{~min}$ after dosing (Fig. 3C).

VAS Score for Scent and Sweetness of ODTs The VAS scores for scent and sweetness are listed in Table 3. The VAS score for scent of Ch2.5-ODTs, Ch5-ODTs, and Ch10-ODTs were significantly higher than those of Cont-ODTs both during disintegration and at $1 \mathrm{~min}$ after dosing. At $5 \mathrm{~min}$ after dosing, the VAS scores returned to the value of the Cont-ODTs.

During disintegration, the VAS scores for sweetness of
Ch2.5-ODTs, Ch5-ODTs, and Ch10-ODTs were higher than those of Cont-ODTs and Ch0-ODTs. While a significant difference was observed between the VAS scores of Ch2.5ODTs, Ch5-ODTs, and Ch10-ODTs and Cont-ODTs, the ContODTs and Ch0-ODTs did not show a significant difference in the VAS scores (Table 3). The VAS scores of all tested ODTs at $1 \mathrm{~min}$ after dosing were significantly higher than those of the Cont-ODTs. The VAS score of Ch10-ODTs was significantly higher than that of Cont-ODTs at $5 \mathrm{~min}$ after dosing.

VAS Score for Overall Palatability of ODTs The VAS scores for overall palatability of Cont-ODT and Ch0-ODT during disintegration were 28.4 and 39.4, respectively, while the difference between ODTs was not significant (Fig. 4A). The VAS scores for overall palatability of Ch2.5-ODTs, Ch5ODTs, and Ch10-ODTs significantly improved to 53.1, 41.1, and 56.2, respectively, compared to that of Cont-ODT. At $1 \mathrm{~min}$ after dosing of ODTs, the VAS scores of Ch0-ODTs, Ch2.5-ODTs, Ch5-ODTs, and Ch10-ODTs were significantly higher than that of Cont-ODTs (Fig. 4B). The difference in the VAS scores remained significant at $5 \mathrm{~min}$ after dosing of 
(A)

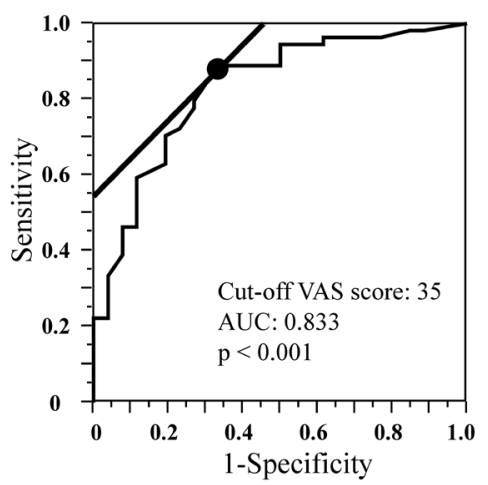

(B)

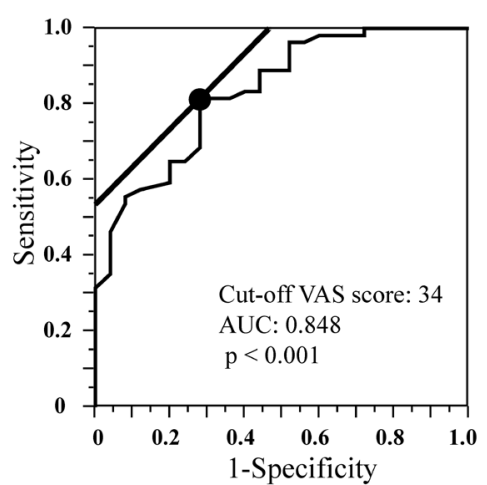

(C)

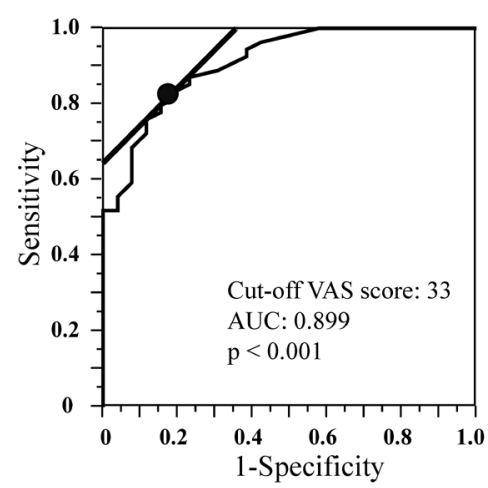

Fig. 5. Receiver Operating Characteristic (ROC) Curve, Cut-Off Visual Analog Scale (VAS) Scores, and AUC During Disintegration (A), at 1 min (B), and $5 \mathrm{~min}(\mathrm{C})$ after Dosing of the Orally Disintegrating Tablets (ODTs)

ROC curve analysis was performed between the VAS scores of overall palatability and the acceptability of the ODTs. The ODTs evaluated of score $1-3$ by volunteers in 5 -point scale in gustatory sensation test were defined as "acceptable." The lines indicate the 45 degrees' straight line, and the cutoff value is indicated by the contact point of the straight line and the ROC curve.

Table 4. Acceptability of Orally Disintegrating Tables (ODTs) Evaluated by 5-Point Scale in Healthy Volunteers

\begin{tabular}{lcccrr}
\hline \hline \multirow{2}{*}{ Evaluation of 5-point scale } & \multicolumn{4}{c}{ Number of volunteers } \\
\cline { 2 - 6 } & Cont-ODT & Ch0-ODT & Ch2.5-ODT & Ch5-ODT & Ch10-ODT \\
\hline 1. Extremely Accepted & 0 & 2 & 3 & 0 & 2 \\
2. Accepted & 5 & 9 & 13 & 6 & 14 \\
3. Neither & 7 & 10 & 7 & 9 & 4 \\
4. Unacceptable & 15 & 2 & 0 & 0 & 1 \\
5. Extremely Unacceptable & 3 & 5 & 7 & \\
\hline
\end{tabular}

Data are shown as the number of volunteers $(n=30)$. The 5 -point scale scores of evaluating acceptability for Cont-ODT, Ch0-ODT, Ch2.5-ODT, Ch5-ODT, and Ch10-ODT were evaluated after the third evaluation using the following items (1, extremely accepted; 2 , accepted; 3 , neither; 4 , unacceptable; 5 , extremely unacceptable). Cont-ODTs, rebamipide orally disintegrating tablets without cocoa powder and sweetener; Ch0-ODTs, Ch2.5-ODTs, Ch5-ODTs, and Ch10-ODTs, rebamipide ODTs prepared by adding a sweetener and $0,2.5,5$, and $10 \%$ cocoa powder, respectively.

ODTs, except for that of Ch5-ODTs (Fig. 4C).

Acceptability of ODTs and ROC of VAS Scores The acceptability of ODTs evaluated using a 5-point scale by the volunteers is shown in Table 4. The results indicated that the Ch2.5-ODTs and Ch10-ODTs were the most acceptable among all the ODTs tested examined. The VAS scores for overall palatability at which the subjects judged acceptability (score 1-3) were used to calculate cut-off scores; three measurements were used to calculate the cut-off scores using ROC analysis (Fig. 5). The cut-off VAS scores for overall palatability at which the subject judged acceptability were 35,34 , and 33 . The $A U C$ values of the analysis were $0.833(p<0.001), 0.848$ $(p<0.001)$, and $0.899(p<0.001)$, respectively.

\section{Discussion}

We aimed to prepare and evaluate ODTs of rebamipide, a therapeutic drug for gastric ulcers with a persistent bitter taste. We prepared rebamipide ODTs using cocoa powder as a taste-masking agent (rebamipide chocolet). Then, we evaluated these ODTs in a gustatory sensation test in human volunteers.

We examined the tablet characteristics of ODTs. The addition of cocoa powder to prepare rebamipide ODTs necessitated an increase in the tableting pressure to achieve the target hardness of $50 \mathrm{~N}$. All formulations achieved the target hardness. Although a tablet hardness of $>30 \mathrm{~N}^{14)}$ is required to withstand the shock during packaging and transport, and a tablet hardness of $>50 \mathrm{~N}$ is preferable in clinical settings. We prepared ODTs with a hardness of $50 \mathrm{~N}$ for clinical use. Rebamipide chocolet showed an increase in the in vitro disintegration time with an increase in the amount of cocoa powder. Ch10-ODTs, containing the highest amount of cocoa powder showed a long in vitro disintegration time; however, the disintegration time was within $30 \mathrm{~s}$, which is the disintegration time set as the standard by the U.S. Food and Drug Administration (FDA $)^{15)}$; and Ch10-ODTs showed good disintegration properties. Disintegration time of ODTs increased as increasing of amount of cocoa powder. The reason of delayed disintegration time for ODTs with higher content of cocoa powder is unclear, however the high lipophilicity of cocoa powder and high tableting pressure for these ODTs appear to take part in the increased disintegration times. No difference was observed in the dissolution properties of the experimental ODTs and the commercially available product. Our results indicate that the addition of cocoa powder to ODTs affected the physical properties of the tablet, but enabled the preparation of rebamipide chocolet having the specified tablet characteristics.

The protocol of this trial was created assuming the actual patient taking ODTs, and palatability was evaluated at three times using the VAS ${ }^{16,17)}$ to evaluate change in palatability over time (Fig. 1). The first evaluation was performed while the ODTs disintegrated in the oral cavity, and the second evaluation was performed immediately after spitting the 
ODTs following $1 \mathrm{~min}$ after consumption, assuming the time required to swallow the tablets "2nd evaluation." Unpleasant palatability for even several minutes after taking ODTs is a major issue in the development of some ODT products. Thus, in our gustatory sensation test, the third evaluation of palatability was performed $5 \mathrm{~min}$ after the subject took the tablet.

The VAS score for bitterness of Cont-ODT at $1 \mathrm{~min}$ after dosing was higher than that during disintegration, and bitterness persisted even after $5 \mathrm{~min}$. This finding indicated that the bitterness of rebamipide enhances or persists after swallowing the ODT rather than during disintegration in the oral cavity. The VAS score for bitterness of Ch0-ODTs (sweetener only) during disintegration showed almost the same as the score of Cont-ODTs. Rebamipide chocolet showed an improvement in the VAS scores during disintegration. In particular, the VAS scores of Ch2.5-ODTs and Ch10-ODTs showed a significant improvement. Compared to Cont-ODT, rebamipide chocolet showed a decreasing trend of VAS scores for bitterness 1 min after dosing, regardless of the amount of cocoa powder. A similar trend of VAS scores was observed even at $5 \mathrm{~min}$ after taking the ODTs (Fig. 3). Formulations containing both cocoa powder and sweetener (Ch2.5-ODTs, Ch5-ODTs, and Ch10-ODTs) showed a marked improvement of overall palatability during disintegration, whereas Ch0-ODT, containing a sweetener alone, showed no significant improvement. The mean VAS score of Ch5-ODTs appeared lower than that of Ch2.5-ODTs, and increase of VAS score depended on amount of cocoa powder was not observed. However, interindividual difference between these ODTs was large, and statistically difference in the VAS scores between Ch2.5-ODTs and Ch5ODTs were not shown. The overall palatability of formulations containing a sweetener and cocoa powder showed improvement even at 5 min after consuming the ODT.

Cocoa powder is typically used for masking the bitterness of foods and medicines. The mechanism underlying the bitterness-masking effect of cocoa powder has been thought that cocoa butter, an oily component, melts because of the heat in the oral cavity, and subsequently, binds to the lipophilic receptors in the taste buds thus inhibiting the binding of other bitter substances. In addition, the odor molecules that spread to the oral cavity evaporate and reach the nasal cavity are said to be largely involved in the taste, and the scent plays a role in reducing the bitterness. ${ }^{18)}$ Kondo et al. use an electronic nose sensor to detect the scent of cocoa powder and evaluate its effect on the bitterness. ${ }^{19)}$ We believe that the peculiar and strong scent of the cocoa powder plays an important role in masking the bitterness. Thus, our results indicated that the bitterness and overall palatability of rebamipide ODTs during disintegration in the oral cavity could be improved by the addition of cocoa powder and sweeter rather than using a sweetener alone. These results indicate that cocoa powder can be used as a bitterness-masking agent to prepare ODTs of other bitter-tasting medicines.

In this study, we used aspartame and thaumatin as sweeteners for rebamipide ODTs. ODTs containing sweeteners alone (Ch0-ODTs) showed no significant decrease in bitterness and improvement in overall palatability during disintegration, whereas its effective taste-masking was observed after 1 and $5 \mathrm{~min}$ following dosing. The difference in the time for the taste-masking effect is dependent on the characteristics of sweeteners. Aspartame and thaumatin are generally con- sidered to take time to develop the masking effect and show a sustained effect. Thus, cocoa powder was necessary to mask the bitterness and improve the palatability of rebamipide ODTs during disintegration. The difference in the timedependent action of masking agents should be considered for developing formulations of ODTs.

The VAS used in our study is known a reproducible method to analyze sensations such as pain. The VAS is sensitive and useful in relative evaluation because it is represented by a numerical score from 0 to 100 . However, the VAS cannot be used for absolute evaluations such as "acceptable dosage of ODTs" or "unacceptable dosage of ODTs." Therefore, in this study, we analyzed the relevance between VAS evaluation and 5-point score of acceptability by performing ROC analysis. We can calculate the cut-off VAS score felt by subjects as "unacceptable" ODTs (scores of 4 or above) by performing ROC analysis. At the during disintegration of the ODT, $1 \mathrm{~min}$ after dosing, and $5 \mathrm{~min}$ after dosing, the cut-off value for "acceptable" for VAS score of overall palatability was 35,34 , and 33 , respectively. That is, if the VAS score of overall palatability is not less than the cut-off value at all evaluation timings, we can infer that the subject has judged the ODT as "acceptable." Comparison of the cut-off values and the VAS score for overall palatability indicated that rebamipide chocolets showed VAS scores higher than the cut-off value for acceptability at all times of evaluation. Thus, the rebamipide chocolet prepared in this study was considered to be an "accepted" formulation with a pleasant feeling after administration.

\section{Conclusion}

We used cocoa powder as a new taste-masking agent for ODTs and prepared ODTs with good dosing properties using rebamipide, which has a persistent strong bitter taste, as the active drug. We named the ODTs containing cocoa powder as chocolet. The evaluation of clinical palatability by healthy subjects using a VAS indicated that bitterness and overall palatability during disintegration, which did not sufficiently improve by the addition of a sweetener alone, was markedly improved the addition of cocoa powder. ROC analysis indicated that the VAS score of overall palatability was not less than the cut-off value at all timings of during disintegration, $1 \mathrm{~min}$ after dosing, and $5 \mathrm{~min}$ after dosing. The formulation was considered to be acceptable. Our results indicate that rebamipide chocolet with a pleasant mouth feel could be prepared using a sweetener and cocoa powder as new taste-masking agent.

Our results suggested the usefulness of organoleptic properties of cocoa powder as a taste-masking agent in ODTs. We believe that cocoa powder can be used as a bitterness-masking agent to prepare ODTs using other active drugs, and thus improve medication adherence of patients.

Acknowledgments The authors are grateful to $\mathrm{Mr}$. Takuya Murao for his excellent technical assistance. We would also like to thank Dr. Shogo Hiraoka (Otsuka Pharmaceutical Co., Ltd.) for his valuable suggestions in developing these formulations.

Conflict of Interest S.U. and N.N. received research grants from Kissei Pharmaceutical Co., Ltd., Takeda Consumer Healthcare Co., Ltd. (Tokyo, Japan), and Otsuka Pharmaceutical Co., Ltd. (Tokyo, Japan). N.N. serves as a consultant 
to Kissei Pharmaceutical Co., Ltd., Otsuka Pharmaceutical Co., Ltd., and Shiseido Japan Co., Ltd. (Tokyo, Japan). None of the authors have conflicts of interest to declare in regard to this study.

\section{References}

1) Strachan I., Greener M., Pharm. Pract., 15, $411-414$ (2005).

2) Steffensen G. K., Pachaï A., Pedersen S. E., Ugeskr. Laeger, 160, 2249-2252 (1998).

3) Hansen D. L., Tulinius D., Hansen E. H., Pharm. World Sci., 30, 65-69 (2008).

4) Polaha J., Dalton W. T. 3rd, Lancaster B. M., South. Med. J., 101, 1106-1112 (2008).

5) Namiki N., Yakugaku Zasshi., 135, 237-243 (2015).

6) Parkash V., Maan S., Deepika, Yadav S. K., Hemlata, Jogpal V., J. Adv. Pharm. Technol Res., 2, 223-235 (2011).

7) Lewis S. C., Langman M. J., Laporte J. R., Matthews J. N., Rawlins M. D., Wiholm B. E., Br. J. Clin. Pharmacol., 54, 320-326 (2002).

8) Wolfe M. M., Lichtenstein D. R., Singh G., N. Engl. J. Med., 340, 1888-1899 (1999).
9) Scheiman J. M., Drugs, 66 (Suppl. 1), 15-21, discussion, 29-33 (2006).

10) Derry S., Loke Y. K., BMJ, 321, 1183-1187 (2000).

11) Nakano Y., Maeda A., Uchida S., Namiki N., Int. J. Pharm., 446, 160-165 (2013).

12) Matsui R., Uchida S., Namiki N., Biol. Pharm. Bull., 38, 17-22 (2015).

13) Uchida S., Yoshita T., Namiki N., Int. J. Pharm., 446, 1-5 (2013).

14) Koseki T., Onishi H., Takahashi Y., Uchida M., Machida Y., Chem. Pharm. Bull., 56, 1384-1388 (2008).

15) Food and Drug Administration, Center for Drug Evaluation and Research (CDER), "Guidance for Industry: Orally Disintegrating Tablets," U.S. Department of Health and Human Services, U.S.A., December, 2008.

16) Carlsson A. M., Pain, 16, 87-101 (1983).

17) Sugiura T., Uchida S., Namiki N., Chem. Pharm. Bull., 60, 315-319 (2012)

18) Heilmann S., Hummel T., Behav. Neurosci., 118, $412-419$ (2004).

19) Kondo C., Fukuoka E., Sasaki T., Namiki N., Takano H., Yasumuro O., Yamamoto T., J. Pharm. Sci. Technol. Jpn., 67, 347-355 (2007). 